\title{
Appropriarsi: transitivo o intransitivo?
}

\author{
Raffaella Setti
}

PUBBLICATO: 28 GENNAIO 2020

\section{Quesito:}

Un nostro lettore contesta la costruzione invalsa nell'uso appropriarsi di qualcosa in luogo di appropriarsi qualcosa.

\section{Appropriarsi: transitivo o intransitivo?}

I

1 verbo appropriarsi entra in italiano nella forma attiva transitiva appropriare ('fare proprio') tra Duecento e Trecento (le prime attestazioni sono di Dino Compagni, Dante, Villani), ma già in questi primi impieghi, che pure mantengono una forma transitiva (quindi con oggetto diretto), lo troviamo accompagnato dalla particella pronominale: "Costoro s'appropriano tutti gli onori" (Compagni); "Perché tu vegghi con quanta ragione / si move contr'al sacrosanto segno / e chi 'l s'appropria e chi a lui s'oppone" (Dante); "La nave col suo tesoro... arrivò in Ispagna, e il re Pietro s'appropriò il tesoro" (Villani).

Se guardiamo i dizionari storici possiamo notare che il verbo, nella sua forma pronominale, non è registrato nel Vocabolario della Crusca (in forma attiva è invece presente fin dalla prima edizione nella variante appropiare) anche se lo si ritrova in alcune definizioni: in particolare nella III edizione (I69I) s.v. mettere per l'espressione mettere sotto che viene spiegata nel suo significato figurato con "Dicesi figurat. Dell'Approfittarsi segretamente, e dello appropriarsi industriosamente che che sia"; nella IV edizione (I729-I738) lo troviamo s.v. mano per spiegare la locuzione mettere le mani innanzi a chicchessia ("vale appropriarsi l'altrui diritto") e s.v. rubare (riferito a un gioco di carte), "è il pigliare o appropriarsi alcune carte di quelle, che s'alzano, o che rimangono dopo essersi distribuite a ciascuno de' giuocatori le sue". La voce autonoma del verbo viene accolta nella V edizione della Crusca (I863I923) sempre nella forma attiva e transitiva appropriare e ancora nutrita di esempi che prevedono l'uso delle forme del verbo con la particella pronominale. E lo stesso trattamento lo ritroviamo nel Tommaseo-Bellini (I86I-I879), che mette a lemma solo la forma appropriare, ma poi snocciola una lunga serie di esempi con la forma pronominale (ancora però transitiva): "T. S'approprió $i$ beni che amministrava. T. Appropriarsi un podere, un diritto, una facoltà. T. Appropriarsi unopera altrui, sia d'ingegno, sia d'arte, Spacciarla per propria. T. Appropriarsi una scoperta. T.I meriti altrui. T. Appropriarsi innocentemente e virtuosamente il merito d'un fatto o d'una intenzione altrui congiungendovisi collopera e col desiderio". Anche il più recente GDLI sceglie di registrare a lemma la forma appropriare "tr. con particella pronominale", salvo precisare "oggi più comunemente intransitivo". Il primo vocabolario che inserisce a lemma il verbo nella forma intransitiva pronominale appropriarsi è lo

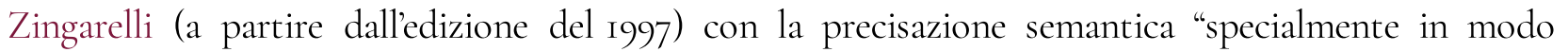
illecito".

Già da questa breve rassegna è evidente che le forme pronominali del verbo, seppur costruito transitivamente, sono sempre state presenti negli usi, da quelli antichi e letterari fino a quelli ottocenteschi della lingua comune riportati da Tommaseo. Nel corso dell'Ottocento però si è affermato il costrutto indiretto (appropriarsi di) a partire da testi giuridici da quanto risulta dalla consultazione di Google libri (al I9/II/2019): il primo esempio giuridico, del I825, è il saggio Esposizione 
di una nuova teoria sulla rendita della terra di Francesco Fuoco, in cui si dice "quindi non si può nè anche dire che l'appropriarsi di un fondo porti seco necessariamente l'appropropriarsi [sic] d'una rendita" (p. 5o); molto significativo che, nello stesso anno, il costrutto compaia nel Vocabolario legale compilato dal dott. Girolamo Sacchetti s.v. Danno dato: "Non si può procedere con l'azione di danno dato contro il colono parziario, che omette di far delle cose senza dolo, e senza l'animo d'appropriarsi di oggetti, che si trovano nel podere da esso lavorati”. Ma una testimonianza che ci conferma la diffusione della forma intransitiva nella prima metà dell'Ottocento è la reazione risentita di Filippo Ugolini che nel suo Vocabolario di parole e modi errati che sono comunemente in uso (I848) cosi si pronuncia: "appropriarsi di una cosa fuggirai di dire in luogo di appropriarsi una cosa".

Il passaggio da transitivo a intransitivo è però avvenuto, molto probabilmente anche per analogia con altri verbi pronominali di significato affine come impossessarsi, impadronirsi, tutti costruiti con la preposizione di. La tendenza conservativa e letteraria dei dizionari che continuano, fino a quasi tutto il Novecento, a registrare il verbo nella forma attiva appropriare transitivo (anche se usato poi nelle forme pronominali) non rende conto dell'uso attuale che troviamo adeguatamente descritto (dopo l'ingresso nello Zingarelli 1997) solo in dizionari decisamente recenti: Vocabolario Treccani, s.v. appropriare "Con questo sign. è ant.; oggi si usa quasi esclusivam. nella forma intr. pron. appropriarsi, cioè impossessarsi, impadronirsi di qualcosa che è di altri o che comunque non spetta: a. di un diritto, di un titolo, di un bene (meno com. a. un diritto, un titolo, un bene); e poi il GRADIT che attribuisce la marca di obsoleto ad appropriare e indica come unica forma in uso appropriarsi intransitivo pronominale. Anche il Sabatini-Coletti registra solo appropriarsi (intr. pron. con arg. ind.) e con lui tutte le edizioni recenti dei principali dizionari dell'uso.

Benissimo quindi ricostruire come si è formato il verbo e comprenderne il significato originario di 'rendere proprio a sé qualcosa', ma nell'uso della lingua bisogna anche sempre considerare le trasformazioni e gli slittamenti che le parole hanno subito nel corso dei secoli e scegliere le forme condivise dalla comunità dei parlanti. Continuare a utilizzare appropriarsi transitivo considerando scorretta la costruzione indiretta, sarebbe come ostinarsi a pronunciare valuto (invece del corrente váluto) o, scegliendo un altro verbo che anticamente era transitivo e ha avuto un esito analogo, dire "non abusare la mia pazienza" invece che "della mia pazienza" (sulla possibilità dell'uso della forma passiva di abusare, in essere abusato, si vedano le osservazioni di Vittorio Coletti).

\section{Cita come:}

Raffaella Setti, Appropriarsi: transitivo o intransitivo? , "Italiano digitale", 2020, XII, 2020/1 (gennaiomarzo)

DOI: $10.35948 / 2532-9006 / 2020.3190$

Copyright 2020 Accademia della Crusca

Pubblicato con licenza creative commons CC BY-NC-ND 